\title{
Hacia una deconstrucción de la unión mujer-madre
}

\section{María Laura Giallorenzi ${ }^{1}$ \\ Consejo Nacional de Investigaciones Científicas Universidad Nacional de Rosario}

\section{Ensayo}

Material original autorizado para su primera publicación en Journal de Ciencias Sociales, Revista Académica de la Facultad de Ciencias Sociales de la Universidad de Palermo.

Recepción:13-02-2020

Aceptación: 30-03-2020

Resumen: Este trabajo propone un abordaje sobre la maternidad basado en los campos disciplinares de la filosofía, la sociología, la historia y la psicología, así como de los estudios sobre género y las teorías desarrolladas por los feminismos. En este sentido, en un primer apartado, se busca definir y caracterizar a la maternidad, poniendo en discusión ciertos enfoques que, desde diversas perspectivas, han centrado su mirada crítica en la asociación unívoca entre mujer y maternidad y la reducción de la feminidad a la maternidad que se ha ido construyendo a lo largo de la historia. En un segundo apartado, se plantea como alrededor de la maternidad se gestan algunas discursividades y operaciones culturales que tienden a perpetuar la unión mujer-madre. Para dar lugar al desentrañamiento de dichas operaciones culturales, se profundiza en la construcción socio-política de la maternidad, y con ella de las categorías de: maternalizacion de las mujeres, maternazgo y maternalismo. Este análisis, permite situar cómo se fueron gestando diferentes sistemas de legitimación de la maternidad como destino para todas las mujeres. El objetivo de pensar y conceptualizar a la maternidad bajo estas lecturas críticas, está dado en buscar, que la maternidad signifique también una experiencia de emancipación para las mujeres y un espacio por el cual obtener los derechos necesarios para ejercer dicho rol.

Palabras claves: maternidad; mujer; asociación mujer-madre.

\footnotetext{
${ }^{1}$ Becaria Doctoral de CONICET, Doctorado en Ciencias Sociales (UBA), Magister en Estudios Culturales (UNR), Licenciada en Sociología (UCES), Profesora Universitaria (UCEL). Profesora titular en la Universidad de Concepción del Uruguay sede Rosario y adscripta en la Facultad de Ciencias Políticas y Relaciones Internacionales de la Universidad Nacional de Rosario. Correo electrónico: giallorenziml@yahoo.com.ar
} 


\section{Towards a deconstruction of the woman-mother union}

Abstract: This work proposes an approach on motherhood based on the disciplinary fields of philosophy, sociology, history and psychology, as well as studies on gender and theories developed by feminisms. In this sense, in a first section, we seek to define and characterize motherhood, discussing certain approaches that, from different perspectives, have focused their critical view on the univocal association between women and motherhood and the reduction of femininity to motherhood that has been built throughout history. In a second section, we stated how around the maternity some cultural discursivities and operations that tend to perpetuate the woman-mother union are formed. To encourage the unravelling of these cultural operations, the socio-political construction of motherhood is deepened, and with it the categories of: women's maternalization, maternalization and maternalism. This analysis allows us to determine how different systems of legitimacy of motherhood were created as a destination for all women. The objective of thinking and conceptualizing motherhood under these critical reading is to seek that motherhood also means an experience of emancipation for women and a space to obtain the necessary rights to exercise this role.

Keywords: maternity; woman; woman-mother association.

\section{Introducción}

Este trabajo reconoce múltiples antecedentes provenientes de los campos disciplinares de la filosofía, la sociología, la historia y la psicología, así como de los estudios sobre género y las teorías desarrolladas por los feminismos.

De este modo, los aportes teóricos revisados para este escrito conceptualizan las nociones de familia y de maternidad en clave sociológica y en perspectiva histórica, basándose en aquellas conceptualizaciones que permitan una mirada crítica acerca de la unión mujer-madre que ha tenido lugar en la cultura hegemónica occidental.

Este ensayo tiene como objetivo discutir acerca de la construcción que se ha realizado a lo largo de la historia sobre el binomio mujer-madre. Desnaturalizando dicha asociación como algo que proviene del orden de lo natural, biológico y/o instintivo, para dar lugar a la visibilización de la existencia de múltiples operaciones culturales, sociales y políticas encargadas de perpetuar, como así, de legitimar el rol de la maternidad como destino obligatorio para todas las mujeres. Para el desarrollo de dicho objetivo, este trabajo se estructura de en dos apartados, explicitados en su contenido a continuación.

En un primer apartado se retoman algunos cuestionamientos claves para desentramar el binomio indiscutido de mujer-madre. Dentro de ellos, la teoría feminista tiene 
un rol fundamental como sustento teórico al permitir revisar críticamente aquellas conceptualizaciones que se gestan alrededor de las mujeres en tanto madres.

En un segundo apartado, con el fin de profundizar acerca de las discursividades tendientes a fortalecer el binomio mujer-madre, se revisa la construcción socio-política de la maternidad que se realiza como política estatal, en lo que se da en llamar maternalizacion de las mujeres. Asimismo, y en este aspecto, se retoman las nociones que se desprenden de dicha construcción, como ser: maternazgo y maternalismo político.

Por último, ya en las conclusiones, se proponen algunas líneas de análisis que permiten ubicar a la maternidad en un plano distinto al que ha sido construido desde el sistema cultural, social y político para las mujeres.

\section{Discutiendo el binomio mujer-madre desde la teoría feminista}

La maternidad no puede ser solamente considerada una ideología y una práctica de control y dominio de las mujeres. Ha sido y es una experiencia de vida y también a partir de ella se intentó exactamente lo opuesto: pelear por los derechos de las mujeres, emanciparlas, liberarlas de la dominación masculina (Nari, 2004, p. 142).

Para desentramar la asociación univoca entre mujer y madre, se retoma la pregunta ya planteada por las teorías feministas: ¿Qué es una mujer? Y, sumándose a éste, otro interrogante crucial: ¿Qué implica ser una mujer?

Revisar estos cuestionamientos y sus problematizaciones desde la teoría feminista, supone tratar de develar que las respuestas a estas preguntas están basadas en la división sexual del trabajo, y en un orden social y cultural tendiente a sujetar a la mujer a espacios y roles no elegidos libremente, sino arbitrariamente asignados por un conjunto de mandatos que condicionan las decisiones.

En este sentido, se plantea una tercera pregunta: ¿Quién o quiénes pueden decir que es una mujer? "De lo que podemos estar seguras que sí sabemos hasta ahora es qué no somos, hasta el punto de que es a través de nuestra lucha que obtendremos la fuerza para romper con la identidad que se nos ha impuesto socialmente" (Federici, 2013, p. 60).

Las primeras preguntas planteadas, fueron formuladas, en primer lugar, por de Beauvoir (2012), y posteriormente por Rubin (1986). Según Osborne y Molina Petit (2008), estas autoras estarían de acuerdo en responder a qué es una mujer, con la siguiente tautología:

«es una mujer» si nos estamos refiriendo al sexo a secas (mujer como hembra humana); pero si nos referimos a la «feminidad» —o al género- entonces tendríamos que hablar del producto de unas relaciones que la «domestican» (o en terminología de Beauvoir, que la convierten en «lo otro») (p. 152). 
Dentro de este planteamiento, de Beauvoir (2012) plantea que la mujer tiene condiciones singulares en relación a la biología como poseer ovarios y un útero que la dejan atada en su subjetividad. En cambio, el hombre/varón olvida sus condiciones anatómicas en su constitución como ser en el mundo, definiéndolas de manera objetiva.

Estos datos biológicos son de suma importancia: representan, en la historia de la mujer, un papel de primer orden; son elemento esencial de su situación: en todas nuestras descripciones ulteriores tendremos que referirnos a ellos. Porque, siendo el cuerpo el instrumento de nuestro asidero en el mundo, este se presenta de manera muy distinta según que sea asido de un modo u otro. Por esa razón los hemos estudiado tan extensamente; constituyen una de las claves que permiten comprender a la mujer. Pero lo que rechazamos es la idea de que constituyan para ella un destino petrificado. No bastan para definir una jerarquía de los sexos; no explican por qué la mujer es lo Otro; no la condenan a conservar eternamente ese papel subordinado (de Beauvoir, 2012, p. 43).

El peso que tiene lo biológico en la constitución de la mujer y del hombre es marcadamente desigual. La mujer ha quedado históricamente atada a su destino biológico en su condición de ser un cuerpo gestante y, en función de ello, una futura madre. Si continuamos con el pensamiento beauvoriano, se puede afirmar que, históricamente, siendo madre la mujer cumple su destino biológico, pero como dice la autora y también las luchas feministas se han encargado de visibilizar, la reproducción y la posibilidad de ser madres de las mujeres ya no están abandonadas a la naturaleza y al azar biológico, sino que se encuentran controladas por la voluntad.

En este mismo sentido, la cultura patriarcal -situada en un sistema capitalista- se ha encargado de fijar fronteras entre lo privado y lo público. Asignando a las mujeres y a éstas en tanto madres, al espacio de lo privado, de lo doméstico, espacio que quedaría desvalorizado en relación a lo que se pone en juego por los hombres en la esfera de lo público.

En este sentido, la maternidad se constituye a lo largo de la historia como el elemento fundante de la feminidad, como aspecto que permite reafirmar que se es mujer. $Y$ en palabras de Osborne y Molina Petit (2008), "no todo ser humano hembra es necesariamente una mujer; necesita participar de esta realidad misteriosa y amenazada que es la feminidad" (p. 157).

\footnotetext{
${ }^{2}$ Es conveniente aclarar brevemente que, en el presente texto, se elige utilizar la categoría teórica de hombre en lugar de la de varón por dos motivos. En principio, porque este ensayo está basado en algunas obras de la teoría feminista que han utilizado en sus traducciones la noción de hombre. Y, en segundo lugar, para desentrañar la idea de que detrás de la categoría hombre, se esconde un universal inclusivo de todos los sujetos que componen una sociedad. La teoría feminista ha demostrado que, a lo largo de la historia, cuando se utilizaba la noción hombre, en general se hacía mención a lo masculino.
} 
Luego, en las teorías feministas dedicadas a teorizar la maternidad desde una visión crítica, aparecen definiciones ligadas a la construcción social, cultural y política, la cual varía en función de las condiciones de la sociedad en la que se encuentre cada mujer. Sin embargo, y para llegar a este tipo de concepción en torno a la maternidad, a lo largo de la historia las mujeres han ocupado posiciones más esencialistas en su condición de madres y cuidadoras según lo que la sociedad valora como bueno o malo para ellas.

En este sentido, Tarducci (2008, p. 11) plantea que la maternidad:

(...) ha sido la institución más esencializada, al punto de confundírsela con la femineidad y hacerla aparecer ligada a principios universales, cuando lo que demuestran la enorme cantidad de investigaciones sobre el tema es que la maternidad es vivida como "natural" sólo por las mujeres que comparten los valores dominantes acerca de lo que tiene que ser una madre. Cuando no se cumplen con las reglas, de clase, de edad, de sexualidad, entre otras, se pone en evidencia que la cuestión es socio-histórica y, por tanto, contingente (las comillas son de la autora).

En esta frase se evidencia la construcción ideologizada de la maternidad asociada a ciertos valores e imágenes que se presentan como obligatorios para la constitución familiar e incluso para los imaginarios en torno a cierto tipo de femineidad. Sin embargo, como plantea Tarducci (2008), existen discursos que cuestionan y que ponen en entredicho tales mandatos, haciendo visible su estrategia de abstracción y habilitando a pensar, definir y experimentar otros modos de maternidad.

Desde la cultura, se difunden discursos tradicionales sobre la maternidad, donde es entendida como mandato, destino ineludible, fuente de felicidad, acto de amor y espacio simbólico de realización plena para todas las mujeres.

Los significantes destacados en el párrafo anterior respecto a ciertos tópicos dominantes en torno a la maternidad son puestos en tensión por las teorías feministas, a través de un recorrido por diferentes aportes que se han preguntado sobre los roles de género $\mathrm{y}$, en particular, han analizado de manera crítica las construcciones y los efectos de los diversos discursos sociales sobre las mujeres, entre los que se destacan los sentidos asociados a la familia y a la maternidad en el sistema de dominación patriarcal (Badinter, 1991; Millett, 1995; de Beauvoir, 2012; De Las Heras Aguilera, 2009, entre otras).

Ciertamente, la crítica feminista a los modos hegemónicos de producir y comprender la maternidad -y por ende, a la idea de femineidad y a la de familia- ha puesto de relieve de una manera irrefutable cómo los discursos sociales construyen y reproducen ciertas definiciones apoyadas en las marcas biológicas que se basan en una estricta separación de las esferas de actuación de varones y mujeres, justificando los roles que desempeñan tanto 
en las estructuras sociales como en el orden simbólico que las produce y reproduce (Gallagher y Lacquer, 1987; Greenfield y Barash, 1999).

En contraposición con las definiciones más tradicionales que se han ocupado de otorgar sentido a la idea de maternidad como parte de la esencia femenina o experiencia natural propia de la hembra humana -que implica, según distintas perspectivas, acciones exclusivas asociadas al instinto, la obligación terrena, el destino divino o el amor (Lagarde, 1994)-, las interpretaciones feministas a las que aquí aludimos, definen a la maternidad como una construcción cultural que se ha transformado y/o fraguado a lo largo de la historia.

Para algunas de las autoras que indagamos en este recorrido, la construcción discursiva de la maternidad se vincula con los modos de organización jerárquicamente sexualizada de la sociedad y puede leerse como resultado de un proceso cultural anclado en la binariedad heterosexual del ordenamiento social (De Beauvoir, 2012; Firestone, 1976; Rich, 1986; Rubin, 1986; Badinter, 1991; Saletti Cuesta, 2008, entre otras).

En esta línea, se plantea que la maternidad y su ligazón inmediata con la conformación de la familia moderna, ha sido interiorizada como elemento natural en la vida de las mujeres a partir de un cúmulo de creencias, sentimientos y conductas presumiblemente innatas o constitutivas, que modelan la representación de un ideal de maternidad derivada de la organización patriarcal y heterosexual de las relaciones sociales (Rich, 1986).

Plantear a la maternidad desde su función dentro de la conformación de la familia moderna, requiere, situarla dentro del sistema patriarcal. Para Rich (1986), el patriarcado es

(...) un sistema familiar y social, ideológico y político en el que los hombres -a través de la fuerza, la presión directa, los rituales, la tradición, la ley y el lenguaje, las costumbres, la etiqueta, la educación y la división del trabajo- decide cuál es o no es el papel que las mujeres deben interpretar y en el que las mujeres están en toda circunstancia sometidas al varón (p. 104).

De esta manera, se puede considerar que, en las sociedades patriarcales contemporáneas, la maternidad hunde sus raíces en la representación generalizada de una particular interpretación de las diferencias biológicas que condiciona los espacios y roles atribuidos a las mujeres, en el contexto de la conformación de la familia nuclear (Daich, 2008; Garay, 2008; Kalinsky y Cañete 2010; Tarducci, 2008).

Dentro del reforzamiento de las concepciones más hegemónicas sobre las categorías de la maternidad y la familia, los medios masivos de comunicación han sido, desde comienzos del siglo XX, un actor muy importante. La presencia de modelos de maternidad y familia hegemónicas que se encuentran en la prensa y el cine, así como en la publicidad de la época, formó parte de una operación cultural tendiente a reforzar los sentidos sociales basados en valores tradicionales sobre dichos roles femeninos: madre y esposa. 
Dicho esto, es necesario también situar a los medios de comunicación como un campo que se encuentra en disputa, dado que en su función de formadores de opinión pública y productores de imaginarios también transmiten significantes considerados contra hegemónicos en cuanto que son elementos diacríticos de la cultura (Fernández Hasan, 2006).

En este sentido, y en relación con la maternidad, se puede observar cómo en la actualidad conviven significantes diversos, que tienden a la producción de nuevas configuraciones mediáticas y discursivas sobre las distintas formas de ejercer la maternidad (Medina Bravo, Figuera Maz y Gómez Puertas, 2013; Valladares Mendoza, 2005).

Esta convivencia de elementos que se constituyen como contradictorios, también se encuentra presente en el conjunto de investigaciones -tanto a nivel internacional como localque estudian el entrecruzamiento entre elementos dominantes y otros disruptivos en torno a la maternidad (Elizalde, 2007; Elizalde y Gutiérrez, 2013; Fernández Fraile, Romo Avilés, Bonaccorsi y Lagunas, 2009; entre otras).

La producción de estos estudios para el caso de Argentina, se ha visto enriquecida a partir de la presencia de activismos políticos relacionados con cierta tradición feminista y de derechos humanos. En este sentido, se observa la presencia de niveles de retroalimentación cada vez más fluidos y más creativos entre la reflexión académica y la militancia sobre estas temáticas, poniendo en el centro de la escena la idea de hacer político lo personal, reproduciéndose en distintos ejes de disputa simbólica, de investigación y de debate político (Pautassi, Arcidiácono y Straschnoy, 2014).

Dentro de esta producción de antecedentes, interesa destacar aquellas investigaciones locales que reflexionan poniendo en cuestionamiento los modos de representación femenina y los roles de género, en clave con los productos culturales, la conformación de las identidades sexo-genéricas, y prácticas que derivan de ellas. Así se puede decir que las maneras de producción cultural de feminidades y masculinidades se encuentran en proceso de redefinición (Cháneton, 2007; Elizalde, 2007; Felitti, 2011; Justo von Lurzer y Spataro, 2015; Justo von Lurzer y Sgandurra, 2015; Laudano, 2010).

En la actualidad, los medios masivos de comunicación, se encuentran mejor situados a partir de su nivel de penetración para construir representaciones hegemónicas en torno a la maternidad y hacerlas circular globalmente. Sin embargo, esto no es excluyente de los medios de comunicación, sino que también es propio de todos aquellos soportes mediáticos encargados de presentar los discursos hegemónicos e ideas dominantes sobre la maternidad (López Diez, 2004).

De esta forma, la importancia de reflexionar sobre los alcances que tienen los discursos de los medios masivos de comunicación en las actuales sociedades mediatizadas 
remite a visualizar cuales son los significados culturales de la maternidad y las representaciones sociales que se hacen de ella. Dentro de este contexto, la maternidad es entendida como:

[el] conjunto de modelos, normas, prescripciones valores, creencias, mitos que se construyen alrededor de la función maternal. Estos medios trasmiten y perpetúan una serie de prácticas y estereotipos relativos a la función maternal, asimismo ejercen una notable influencia en el modelaje del comportamiento de la mujer madre (Valladares Mendoza, 2005, p. 6).

Concretamente, se puede decir que el trato que le dan los medios de comunicación social a la mujer en general, y a la mujer en tanto madre en particular, es reducido y basado en un sistema patriarcal de asignación de tareas según género. Así, se puede identificar a las mujeres como sujetos dependientes de otros -masculinos fundamentalmente- y como seres que tienen las cualidades de ser débiles y románticas, pero es difícil encontrar a la mujer y a la madre representada a partir de elementos como la racionalidad o autonomía (Gallagher, 1979). Interesa remarcar una constante en esto, "un factor intrínseco, prácticamente en toda imagen de la mujer en los medios de comunicación social -si bien tiene características algo diferentes de un país a otro-, es un motivo dicotómico que las define" (Gallagher, 1979, p. 14), ya sea como totalmente buenas o totalmente malas.

De Lauretis (2000) ha profundizado sobre la diferenciación entre mujer y mujeres, definiendo a la mujer como una construcción basada en la ficción de la cultura occidental moderna. Al respecto, López Diez (2004) aclara que:

esta construcción teórica tiene pertinente aplicación en la medida en que la mujer que representan, masiva y mayoritariamente los medios de comunicación (prensa, radio, televisión, internet, las canciones, los video juegos, los comics...) es el producto de las tecnologías de género. Estas tecnologías de género (De Lauretis, 2000) construyen, reproducen, refuerzan y reelaboran continuamente 'la mujer' sobre la base de los arquetipos tradicionales -madre-puta-víctimaobjeto sexual, estatus vicario...-, y muy pocas veces dejan entrar en el guión a otro tipo de mujeres; es decir, a las mujeres reales (López Diez, 2004, p. 8) (la cursiva me pertenece).

En sintonía con la definición del modelo de mujer anteriormente expuesta, se puede afirmar que el modelo ideal de maternidad que los medios masivos de comunicación se encargan se transmitir, coincide con la definición elaborada por Hays (1998) para quien existe la maternidad intensiva como el modelo cultural que se impone en forma reciente, el cual consiste en la madre individual, única, abnegada, de tiempo completo, dedicada e instruida en sus deberes y responsabilidades maternales.

A continuación, se prioriza una de las críticas que se le hace a este ideal de maternidad de la mano de Badinter (1991), quien nos invita a pensar sobre la pérdida de 
libertad para las mujeres, las cuales pierden espacios de autonomía siguiendo las prescripciones que le permiten convertirse en una buena madre. Según esta autora muchas son las instituciones encargadas de transmitir discursos asociados a este ideal de maternidad que propone una crianza natural y una vuelta de las mujeres al hogar. En el fragmento expuesto a continuación se expone - en palabras de Badinter- con claridad este argumento:

Lo que sostengo es que no puede ser que el modelo de la Liga de la Leche se imponga a todas las mujeres. Yo no estoy de ninguna manera contra la lactancia porque sé muy bien que, para algunas mujeres, es una fuente de plenitud y disfrute. Pero no quisiera que este modelo se vuelva obligatorio (Kantor, 2010, p. 1).

Al abordar los sentidos sociales asociados a la maternidad, los estudios sobre comunicación y género, han afirmado que el imaginario materno se compone de dos elementos centrales que lo sostienen: el instinto materno y el amor maternal (Badinter, 1991; Palomar Verea y Suárez de Garay, 2007). Dichos sentidos son transmitidos a través de diversos circuitos discursivos tales como la prensa escrita, televisión, radio, y más recientemente, las redes sociales. Estos circuitos de circulación de información tienden a reforzar estereotipos de género esencialistas, siendo transhistórico y transcultural, y en plena relación con argumentos biologicistas y mitológicos (Palomar Verea y Suárez de Garay, 2007).

La crítica feminista se ha encargado de denunciar la concepción esencialista de la maternidad. Al respecto, Badinter (1991) señala que la maternidad es "como si se tratara de una actividad preformada, automática y necesaria que sólo espera la oportunidad de ejercerse. Como la procreación es natural, nos imaginamos que al fenómeno biológico y fisiológico del embarazo debe corresponder una actitud maternal determinada" (p. 1).

En este sentido, "la maternidad se puede ver como el conjunto de prácticas discursivas a través de las cuales se ponen en práctica las elaboraciones culturales de la diferencia sexual y la reproducción biológica" (Palomar Verea y Suárez de Garay, 2007, p. 315).

En síntesis, es posible decir que alrededor de la maternidad se gestan algunas discursividades y operaciones culturales que tienden a perpetuar la unión mujer-madre, como así también una forma de ejercer este rol. Ahora bien, ¿en qué consiste la construcción que se realiza en esta asociación mujer-madre?

\section{La construcción socio-política de la maternidad}

Para trabajar la construcción socio-política de la maternidad, es necesario interrogar las categorías que se desprenden de ella, tales como la maternalizacion de las mujeres, el 
maternazgo y el maternalismo. De este modo, se puede situar cómo se fueron gestando diferentes sistemas de legitimación de la maternidad como destino para todas las mujeres (Mojzuk, 2014; Nari, 2004).

La maternalización de las mujeres (es decir la progresiva confusión entre mujer y madre, feminidad y maternidad) se fue construyendo y extendiendo gradualmente en diferentes ámbitos y planos de la vida social, del mercado de trabajo, de las ideas y prácticas científicas y políticas (Nari, 2004, p. 101).

Esta construcción se fue haciendo de manera tal que garantizó tanto aspectos simbólicos como materiales, subsumiendo a la maternidad, todo lo ligado a la feminidad. Mediante esta construcción, ser mujer significa ser una madre.

Varias disciplinas se encargan de sostener este proceso de maternalización de las mujeres, como ser la religión y la psicología, pero también diferentes especialidades y ramas de la ciencia médica, como la obstetricia, la ginecología y la puericultura. Desde ellas se constituye el lugar desde donde emanan las justificaciones y legitimaciones que se postulan como dogmas. "La maternidad estaba inscripta en la naturaleza femenina, en los cuerpos de las mujeres, en su biología. La maternalización no implicaba algo obvio, que las mujeres podrían ser madres, sino que solo debían ser madres" (Nari, 2004, p. 101).

En este mismo sentido, se le crea la obligación a la mujer de ser madre, pero no solamente ello, sino que dicha práctica debe estar ejercida desde sentimientos y formas de ser que también se han encargado de definir ciertas disciplinas, como la psicología y la psiquiatría. El objetivo de todo esto, "era construir una relación indisoluble entre madre y niño que constituyera el eje articulador de la familia moderna” (Nari, 2004 p. 102).

La maternalización de las mujeres se sustenta únicamente en la capacidad reproductiva de las mujeres. De esta forma, la maternidad queda atada a la biologización de este rol, sin tener en cuenta que ser madre para una mujer puede comprender mucho más que gestar y parir. Pensar a la maternidad en estos términos, permite comprender que no se concibe a las mujeres como sujetos de derechos con capacidad de decidir cuándo y cómo ser madres.

Contextualizar este proceso dentro de una política de Estado, lleva a que se hable de maternalismo político. Las políticas referidas a la maternidad (reformas en Códigos Civiles y Penales, leyes sociales y laborales), plantean un conjunto de ideas que se leen en términos de disputas, ya que son muy disímiles en los términos en que definen el lugar para la mujermadre en la sociedad.

Hay sectores que a lo largo de la historia pretenden excluir a las mujeres del mercado laboral, fortaleciendo con ello el papel de la mujer dentro del hogar y de subordinación a los 
intereses de la familia y de la institución del matrimonio. Para ello, deben definir de manera contundente el lugar que le corresponde a la mujer:

\begin{abstract}
Las mujeres deben participar del contrato de matrimonio. Pero el contrato sexual requiere que las mujeres se incorporen a la sociedad civil sobre una base diferente de la de los varones. Los varones crean la sociedad civil patriarcal y el nuevo orden social está estructurado en dos esferas. La esfera privada está separada de la vida pública civil; la esfera privada es y no es parte de la sociedad civil, y las mujeres son y no son parte del orden civil. Las mujeres no son incorporadas como individuos sino como mujeres, lo que en la historia del contrato original significa que participan en tanto subordinados naturales (Pateman, 1995, p. 249-250, según cita Brown, 2007, p. 35).
\end{abstract}

Por otro lado, hay quienes exigen la ampliación de derechos de las mujeres, justamente por su condición de madres. En este terreno de disputas, lo que está en juego además del destino de muchas mujeres, es la definición de maternidad con la que un Estado construye sus políticas sociales en torno a la temática de la maternidad. Y justamente en este ejercicio, las mujeres encontrarán en la maternidad un lugar emancipador desde donde se pueda ejercer el poder.

La maternalización de las mujeres no tuvo únicamente lugar en esta región. Sino que aquí se siguieron las pautas de un proceso de índole más abarcador que incluyó al mundo occidental entre fines del siglo XVIII y principios del XX. Las transformaciones que guiaron este proceso abrieron paso a las sociedades capitalistas y al papel de la familia como espacio clave para que el modo de producción sea rentable.

En la Argentina de principios del siglo XX, gran parte de los derechos conquistados por las mujeres estuvieron vinculados al ejercicio de la maternidad. "Obtuvimos licencias por maternidad varias décadas antes que derechos políticos. El objetivo era proteger la maternidad: las mujeres contribuían como "recursos" mucho antes que como sujetos. "Poblar" el país requería de mujeres que canalizaran la aspiración nacional" (Faur y Vázquez Laba, 2018, párr. 7) (las comillas son de las autoras).

Como sostiene Elizabeth Jelin, las prácticas sociales, entretanto, desplegaban modalidades que discrepaban de las normas legales. Ocurrió con el divorcio, con la patria potestad compartida, con la unión de parejas del mismo sexo y ocurre, de manera persistente, con el aborto. Quizás porque erosionar el mandato de la mujer como madre derriba uno de los pilares más profundamente arraigados en una cultura de la desigualdad entre géneros. Lo que está en juego es el abandono de las ficciones relacionadas con la maternidad, y la necesidad de preservar -por acción u omisión- un modelo de mujer como instrumento de la reproducción social (Faur y Vázquez Laba, 2018, párr. 8).

Plantear el concepto de maternalismo político conlleva a situar a la maternidad entre dos polos que se presumen opuestos: como un asunto privado o un asunto público (Knibiehler, 2001; Palomar Verea, 2005). 
La maternidad tomada como un asunto público, según Nari (2004) "es una tendencia aparentemente contradictoria pero coadyuvante, el Estado politiza la maternidad al convertirla en un objeto de preocupación y debate público y político" (p. 18). Ubicar a la maternidad en este plano, además de posibilitar derechos para las mujeres, implica principalmente el control de las mismas y de su maternidad.

De esta forma, la figura de la niñez y la de la maternidad surgen como objetos de vigilancia y de determinadas tecnologías de poder al interior de la institución familiar. Las madres se convierten también en una figura de tutelaje estatal, aunque no del mismo modo que el menor. El disciplinamiento de las sociedades occidentales le otorga el lugar de objeto de diversas formas de vigilancia y control más o menos sutiles con el fin de promover una manera apropiada para ejercer dicha función social.

"Las pedagogías maternales constituyen una tecnología de género que se ha basado en diferentes recursos discursivos y no discursivos para implantar la separación entre lo apropiado y lo inapropiado" (Darré, 2013, p. 8). Lo inapropiado es una categoría que representa aquellas circunstancias, coyunturas o simples características que históricamente se han definido como problemáticas respecto a un modelo de maternidad hegemónico.

Lo inapropiado con respecto a la maternidad tiene que ver con la imposición del modelo de maternidad intensiva, definida por Hays (1998) como un modelo cultural e históricamente construido a partir de pensar a los niños como seres valiosos e inocentes, donde la crianza debe ser llevada a cabo ante todo por las madres individuales, estar centrada en las necesidades de los niños y niñas a partir de una atención intensiva y donde los métodos de crianza son determinados por expertos. La instauración lenta pero persistente de este modelo, permite construir un sistema de sanciones y recompensas basado en registros morales, criminológicos, médicos, psiquiátricos y psicológicos, que van a indicar en cada época cómo ser una madre apropiada (Darré, 2013, p. 8).

En este sentido, Darré (2013) plantea una pregunta importante: “¿en qué momento la crianza dejó de ser un asunto fácil que cualquier mujer podía llevara adelante para convertirse en un problema complejo y de efectos insospechados, que lleva aparejada la necesidad de conocimientos, aptitudes especiales y conciencia de la complejidad?" (p. 7).

A partir de finales del siglo XIX la crianza de los hijos, como así también la relación madre-hijo/a comenzó a verse interpelada por un conjunto de saberes y de expertos. "Esto se debió, en parte a una valoración utilitaria del niño enmarcada en una concepción de la población como riqueza" (Cernadas Fonsalias, 2014, p. 120). De esta manera, se establecía en esa época que la crianza de los niños, niñas debía estar controlada por los profesionales de la salud, transformando a las madres y sus saberes en un obstáculo en este proceso (Cernadas Fonsalias, 2014). 
La maternidad se ha convertido así en un dispositivo más de control de los sujetos en las sociedades disciplinarias,

inscribiendo en sus cuerpos un modo y una forma de ser. Pero no cualquier manera de ser. Lo que inscriben en el cuerpo son un conjunto de praxis, saberes, instituciones, cuyo objetivo consiste en administrar, gobernar, controlar, orientar, dar un sentido que se supone útil a los comportamientos, gestos y pensamientos de los individuos (García Fanlo, 2011, p. 2).

La manera en que los Estados convierten a la maternidad en un asunto político se ha denominado maternalismo. Esto se da a partir de la existencia de diversas políticas que se definen como pronatalistas o de control de natalidad, entre otras.

Desde fines del siglo XX el maternalismo en este país (principalmente en Buenos Aires) estuvo regulado por algunas instituciones que tenían como fin la corrección de mujeres jóvenes y adultas: la Sociedad de Beneficencia y su Asilo de la Pobreza y del Trabajo, la Casa del Buen Pastor, la Sociedad de San Vicente de Paul (1889), la obra de Don Bosco (1893), las Madres Argentinas (1897), la Liga de Protección de las Jóvenes (1902), la Asociación Escuelas y Patronatos (1906), las Cantinas Maternales (1915), la Casa del Niño (1916), el Club de Madres (1917), y el Instituto de la Maternidad (1921) (Darré, 2013).

Estos y otros espacios operan como verdaderos dispositivos pedagógicos (Darré, 2013), los cuales se orientan a educar a una población de madres que no encuadran sus maternidades dentro lo socialmente aceptado. De esta manera, el dispositivo (Foucault, 1985) define aquello que resulta inapropiado con respecto al modelo de maternidad hegemónico, estableciendo también definiciones sobre la supuesta naturaleza de las madres.

Como se puede ver en el recorrido desplegado, las políticas estatales vinculadas a la institucionalización de la familia y de dos de sus figuras más importantes, varían en relación al suelo político de cada momento histórico, lo cual implica que no existiría una única manera de delinear figuras y funciones como las de la niñez y la maternidad. Así, el análisis del modo en que ellas se configuran, y el estudio de las políticas estatales implementadas en relación a dichas figuras, da cuenta de la estrategia biopolítica que opera como condición de posibilidad para su emergencia y sus eventuales transformaciones. Y, a su vez, todo ello hace visible la racionalidad político-económica que caracteriza la gubernamentalidad de la época, dado que, en cada momento histórico de una sociedad dada, se requiere la institucionalización de ciertas figuras que devienen imprescindibles para el sostenimiento de la forma de gobierno que se pretenda perpetuar desde el Estado.

En otras palabras, del mismo modo que ciertas figuras tales como la familia, la niñez (ya sea tutelada o bien con derechos), la maternidad, etc., son producidas, ciertas estrategias biopolíticas y determinadas formas de disciplinamiento (tales como los dispositivos 
pedagógicos, la difusión de preceptos morales inherentes al discurso médico, el alojamiento de individuos en determinados espacios de tutelaje, etc.), van simultáneamente interpelando, cuestionando y transformando su supuesta naturaleza. Es de esperar entonces que, así como la figura del menor tutelado fue diluyéndose, al ser sustituida por la del niño, niña y adolescente como sujeto de derecho (Bright, 2011; Fernández, 2009), la figura de la maternidad como un único modelo que forma parte de una familia biparental heterosexual, en donde las mujeres tienen la obligación de asumir individualmente el cuidado de los hijos, también se vaya diluyendo.

\section{Conclusiones}

Las páginas que constituyen este ensayo, tuvieron como objetivo un abordaje transdiciplinario de la construcción de la categoría de la maternidad como elemento fundante de la identidad femenina. Se buscó, deconstruir esta unión indiscutida entre mujer y madre, a partir de retomar algunos lineamientos claves de la teoría feminista que permiten una mirada crítica sobre dicha unión.

Discutir acerca de los componentes que le han sido asignados a la función social de la maternidad tales como la noción de instinto, biológico, o su constitución como destino para todas las mujeres que quieran realizarse por completo, ha permitido dar visibilidad al papel que ha tenido a lo largo de la historia el Estado -como ejecutor de políticas destinadas a la población en general y especialmente a las mujeres- y a la cultura como constructores de distintas operaciones destinadas a justificar el binomio mujer-madre.

En este sentido, fue clave para el desarrollo problemático de este trabajo poder situar estas discusiones en el marco del maternalismo político, entendido como el conjunto de acciones que realiza un Estado para llevar adelante un proceso de maternalizacion de todas las mujeres.

Asimismo, y, por último, se plantean algunas líneas que permiten seguir reflexionando sobre la temática. En primer lugar, se destaca que el accionar del Estado en tanto actor fundamental en las operaciones políticas y culturales tendientes a conservar desde lo hegemónico a la maternidad como rol fundamental para todas las mujeres, encuentra en la actualidad algunos puntos confusos. Entre ellos, interesa destacar, la desigualdad existente entre hombres y mujeres acerca de las licencias de maternidad y paternidad, dejando entrever que al parecer el único interés que se tiene en que las mujeres sean madres, es el incremento de la población, y en un contexto económico signado por el capitalismo, el incremento de la mano de obra. 
En este mismo sentido, se plantean algunos interrogantes: ¿Para qué se quiere que las mujeres sean madres si luego de que tienen a sus hijos no se las acompaña?; ¿Para qué se les inculca desde sus primeros años de vida un conjunto de acciones que supuestamente sirven para criar a los hijos, hijas, si luego a los pocos meses el mercado laboral te obliga a retornar al puesto de trabajo?

Las contradicciones en cuanto a afirmar que las mujeres deben ser madres en algún momento de sus vidas, no es algo exclusivo del Estado en tanto agente que legitima dichas creencias, sino que también la cultura y quienes forman parte de ella tienen otro rol necesario para que esto perpetúe a lo largo del tiempo. Así, se plantean algunas preguntas para tratar de visibilizar las incongruencias existentes en dichos mensajes, mitos y creencias. ¿Qué busca una cultura que mediante muchas de sus creencias y costumbres legitima la unión mujer-madre, pero luego no existen espacios que acompañen a dichas personas?; ¿Para qué se les exige socialmente a las mujeres que sean madres, si luego la sociedad es hostil con ellas y sus hijos, hijas recién nacidos?

Así, y para finalizar este escrito, se busca que la maternidad pase a ser una elección personal de cada mujer, y cuando así lo decida pueda ejercer dicho rol en un marco de derechos que acompañen su decisión.

\section{Referencias bibliográficas}

Badinter, E. (1991). ¿Existe el instinto maternal? Historia del amor maternal. Siglos XVII al $X X$. Barcelona: Paidós.

Beauvoir de, S. (2012). Segundo Sexo. Buenos Aires: Debolsillo.

Bright, M. (2011). Miedo a los niños: una reflexión sobre la gestión de los riesgos a través de la intervención biopolítica sobre la niñez pobre. Cátedra Paralela, 8, 151-165. Recuperado de http://www.catedraparalela.com.ar/images/rev_articulos/arti00106f001t1.pdf

Brown, J. (2007). Ciudadanía de mujeres en Argentina. Los derechos (no) reproductivos y sexuales como bisagra, lo público y lo privado puesto en cuestión. Tesis de Maestría en Ciencia Política y Sociología. Obtenida. Facultad Latinoamericana de Ciencias Sociales (Flacso). Recuperada de http://legacy.flacso.org.ar/uploaded_files/Publicaciones/Tesis_Josefina_Leonor_ Brown.pdf

Cernadas Fonsalías, C. (2014). Cuidados en disputa. Las voces de los expertos y de las madres migrantes en el ámbito de la Salud Pública. En M. Tarducci (Comp.), 
Feminismo, Lesbianismo y Maternidad en Argentina (pp. 115-132). Buenos Aires: Librería de Mujeres.

Cháneton, J. (2007). Género, poder y discursos sociales. Enciclopedia Semiológica. Buenos Aires: Eudeba.

Daich, D. (2008). Buena madre. El imaginario maternal en la tramitación judicial del infanticidio. En M. Tarducci (Comp.), Maternidades en el siglo XXI (pp. 61-86). Buenos Aires: Espacio.

Darré, S. (2013). Maternidad y tecnologías de género. Buenos Aires: Katz.

De las Heras Aguilera, S. (2009). Una aproximación a las teorías feministas. Universitas. Revista de Filosofía, Derecho y Política, 9, 45-82. Recuperado de http://universitas.idhbc.es/n09/09-05.pdf

De Lauretis, L. (2000). La tecnología de género. En Diferencias, etapas de un camino a través del feminismo (pp. 33-69). Madrid: Horas y Horas.

Elizalde, S. \& Gutiérrez, M. (2013). Un campo de estudio e intervención en tiempo futuro. Avatares de la comunicación y la cultura, (5), 3-11. Recuperado de http://ppct.caicyt.gov.ar/index.php/avatares/article/viewFile/3154/3017

Elizalde, S. (2007). De encuentros y desencuentros. Hacia un mapa indicial del vínculo género/comunicación. Cuadernos Críticos de Comunicación y Cultura, 3, 15-40. Recuperado de https://nucleodegenerounr.files.wordpress.com/2013/03/elizaldede-encuentros-y-desencuentros-hacia-un-mapa-indicial-del-vinculo-generocomunicacion.pdf

Faur, E. y Vázquez Laba, V. (2018). La maternidad será deseada o no será. Revista Anfibia. Universidad Nacional de San Martín. Recuperado de http://revistaanfibia.com/ensayo/la-maternidad-sera-deseada-o-no-sera/

Federici, S. (2013). Revolución en punto cero. Trabajo doméstico, reproducción y luchas feministas. Madrid: Traficantes de sueños.

Felitti, K. (2011). Madre no hay una sola. Experiencias de la maternidad en la Argentina. Buenos Aires: Ciccus

Fernández, S. (2009). Las políticas de la infancia. Una infancia de la política. Cátedra Paralela, 6, 53-63. Recuperado de http://www.catedraparalela.com.ar/images/rev_articulos/arti00069f001t1.pdf

Fernández Fraile, M.; Romo Avilés, N.; Bonaccorsi, N. y Lagunas, C. (Comps). (2009). Los estudios de las Mujeres de España y Argentina. Propuesta para el debate. Buenos Aires: Prometeo. 
Fernández Hasan, V. (2006). Estudios comunicológicos y Estudios de Género: La relación pública/privado en los medios de comunicación. Quórum Académico, 3(2), 3954. Recuperado de http://www.redalyc.org/pdf/1990/199016789003.pdf

Firestone, S. (1976). La dialéctica del sexo. En defensa de la revolución feminista. Barcelona: Kairos.

Foucault, M. (1985). El juego de Michel Foucault. En Saber y verdad (pp. 127-162). Madrid: Ediciones de la Piqueta.

Gallagher, M. (1979). El modo de presentar a la mujer en los medios y de comunicación social y su participación en ellos. Reino Unido: Instituto de Tecnología de la Educación.

Gallagher, M. y Lacquer, T. (Eds.) (1987). The making of the modern body. Sexuality and society in the nineteenth century. Berkeley: University of California Press.

Garay, R. (2008). El destino de ser madres: la ideología de la maternidad como soporte discursivo de las nuevas tecnologías reproductivas. En M. Tarducci (Comp.), Maternidades en el siglo XXI (pp. 29-60). Buenos Aires: Espacio.

García Fanlo, L. (2011). ¿Qué es un dispositivo? Foucault, Deleuze y Agamben. A Parte Rei. Revista de Filosofía, 74. Recuperado de http://serbal.pntic.mec.es/ cmunoz11/fanlo74.pdf

Greenfield, S. y Barash, C. (1999). Inventing maternity. Politics, science and literature. Lexington: The University of Kentucky Press.

Hays, S. (1998). Las contradicciones culturales de la maternidad. Barcelona: Paidós. Justo Von Lurzer, C. y Sgandurra, F. (2015). Mamá Mala, crónicas de una maternidad inesperada. Buenos Aires: Hekht.

Justo von Lurzer, C. y Spataro, C. (2015). Tontas y víctimas. Paradojas de ciertas posiciones analíticas sobre la cultura de masas. La trama de la Comunicación, 19, 113-129. Recuperado de http://www.latrama.fcpolit.unr.edu.ar/index.php/trama/article/view/517/399

Kalinsky, B. y Cañete, O. (2010). Madres frágiles: un viaje al infanticidio. Buenos Aires: Biblos

Kantor, R. (30 de abril de 2010). Contra la tiranía de la madre perfecta. Revista $\tilde{N}$. Recuperado de http://edant.revistaenie.clarin.com/notas/2010/04/30/_02189856.htm

Knibiehler, Y. (2001). La construction sociale de la maternité, en Maternité, affaire privée, affaire publique. París: Bayard. 
Lagarde, M. (1994). Maternidad, Feminismo y Democracia. En C. Talamante Díaz; F. Salinas Campeas; M. Valenzuela (Comps), Repensar y Revisar la Maternidad (pp. 19-35). México D.F.: Grupo de Educación Popular con Mujeres.

Laudano, C. (2010). Visibilidad mediática de la violencia hacia las mujeres en Argentina (1983-2009). Revista Derecho y Ciencias Sociales, (3), 88-110. Recuperado de http://sedici.unlp.edu.ar/bitstream/handle/10915/15248/Documento_completo.pdf ?sequence $=1$

López Diez, P. (2004). La mujer, las mujeres y el sujeto del feminismo en los medios de comunicación. En Manual de información en género. Madrid: IORTV (RTVE) / Instituto de la Mujer. Recuperado de http://www.pilarlopezdiez.eu/pdf/MujerMujeresSujetoFem.pdf

Medina Bravo, P.; Figuera Maz, M. y Gómez Puertas, L. (2013). El ideal de madre en el siglo XXI. La representación de la maternidad en las revistas de familia. Revista Estudios sobre el Mensaje Periodístico, 20(1), 487-504. Recuperado de http://revistas.ucm.es/index.php/ESMP/article/view/45244/42584

Millett, K. (1995). Política Sexual. Madrid: Cátedra

Mojzuk, M. (2014). Entre el maternalismo y la construcción socio-política de la maternidad. Patagonia Libertaria. Recuperado de https://patagonialibertaria.files.wordpress.com/2014/12/68101129-entre-elmaternalismo-y-la-construccion-socio-politica-de-la-maternidad-marta-mojzuk.pdf

Nari, M. (2004). Políticas de maternidad y maternalismo político: Buenos Aires, 1890-1940. Buenos Aires: Biblos.

Osborne, R. y Molina Petit, C. (2008). Evolución del concepto de género: selección de textos de Beauvoir, Millet, Rubin y Butler. EMPIRIA. Revista de Metodología de las Ciencias Sociales, 15, 147-182. Recuperado de http://espacio.uned.es/fez/eserv/bibliuned:Empiria-2008-15-0006/Documento.pdf

Palomar Verea, C. y Suárez de Garay, M. (2007). Los entretelones de la maternidad. A la luz de las mujeres filicidas. Estudios Sociológicos, 25(74), 309-340. Recuperado de http://www.redalyc.org/articulo.oa?id=59825202

Pautassi, L.; Arcidiácono, P. y Straschnoy, M. (2014). Condicionando el cuidado. La Asignación Universal por Hijo para la Protección Social en Argentina. Íconos. Revista de Ciencias Sociales, (50), 61-75. Recuperado de http://www.redalyc.org/articulo.oa?id=50931716004 
Rich, A. (1986). Nacemos de Mujer: La maternidad como experiencia e institución. Valencia: Cátedra.

Rubin, G. (1986). El tráfico de mujeres: notas sobre la "economía política" del sexo. Revista Nueva Antropología, VII, 30, 95-145. Recuperado de https://www.caladona.org/grups/uploads/2007/05/El\%20trafico\%20de\%20mujere s2.pdf

Saletti Cuesta, L. (2008). Propuestas teóricas feministas en relación al concepto de maternidad. Clepsydra, 7, 169-183. Recuperado de http://publica.webs.ull.es/upload/REV\%20CLEPSYDRA/072008/11\%20Saletti.pdf

Tarducci, M. (Comp.) (2008). Maternidades en el siglo XXI. Buenos Aires: Espacio.

Valladares Mendoza, B. (2005). La maternidad y los medios masivos de comunicación. Un análisis de artículos periodísticos y de propaganda comercial en Costa Rica. Diálogos. Revista Electrónica de Historia, 5(1-2), 1-19. Recuperado de http://www.redalyc.org/pdf/439/43926968019.pdf 